Leading Article

\title{
The pathogenesis and treatment of Graves' ophthalmopathy
}

\author{
I.R. Jones \\ Newcastle General Hospital, Westgate Road, Newcastle upon Tyne NE4 6BE, UK.
}

Graves' ophthalmopathy (GO) is probably an autoimmune condition although the pathogenesis is still not fully understood. GO is frequently, though not invariably, associated with Graves' disease and it may in fact be a separate disease entity. Ophthalmopathy is commonest in the 30 to 50 age group ${ }^{1,2,3}$ and is more common in women $(2: 1$, female:male), although the female preponderance is not so marked as in Graves' disease where the ratio is $6: 1 .^{4,5,6} \mathrm{~A}$ family history of autoimmune thyroid disease is frequently present which may or may not be accompanied by ophthalmopathy. The pattern of inheritance is not a simple one and no single gene appears to be implicated. In Graves' disease it is likely that the genes conferring susceptibility are associated with the major histocompatibility genes and expression of major histocompatibility antigens B8 and DR3 confers an increased risk for Graves' disease.,

The most striking pathological feature of GO is the marked increase in muscle bulk. The eye muscles show lymphocytic infiltration and have increased intercellular spaces with mucopolysaccharide and water. The structure of the muscle is usually normal although there may be swelling of the fibres and loss of striations and in more severe cases fibre atrophy and disordered muscle structure. The retrobulbar connective tissue also contains numerous lymphocytes and fibroblasts and there is proliferation of the connective tissue with newly formed collagen. The spaces between the collagen fibres contain amorphous material, mainly hyaluronic acid and other glycosaminoglycans which increase the osmotic pressure and trap water. The main result of all these changes is an increase in the total orbital contents.

The presence of immune response cells in the orbit, the deposition of immunoglobulins on orbital tissues and the association with autoimmune thyroid disease suggests that the underlying mechanism is an autoimmune process. Cell-mediated immune responses and

Correspondence: I.R. Jones M.D., M.R.C.P.

Received: 19 March 1987 antibodies against eye muscle antigens have been identified but it is not known whether the damage results from cell-mediated immunity, circulating immune complexes or pathogenic antibodies. Cellmediated immunity has been clearly demonstrated with the production of macrophage migration inhibition factor (MIF) by sensitized T lymphocytes in response to retro-orbital antigens using both crude orbital antigens consisting of muscle and $\mathrm{fat}^{8}$ and retro-orbital muscle. ${ }^{9}$ Attempts to purify the antigen from retro-orbital tissue suggested that thyroglobulin or a derivative was responsible, ${ }^{10}$ although there has been no confirmatory evidence for this. $T$ cell subsets have not been studied extensively in GO but two cases with abnormal helper:suppressor $\mathrm{T}$ cell ratios and increased circulating activated $T$ cells have been described. " Lymphocytes from patients with GO have been shown to induce lysis in eye muscle cells ${ }^{12}$ and lymphocytes incubated with fibroblasts provoke an increase in glycosaminoglycans. ${ }^{13}$

Antibodies, termed ophthalmic immunoglobulins, have been described in the serum of patients with GO which bind specifically to eye muscle membranes. ${ }^{14}$ The specific nature of ophthalmic immunoglobulins was shown by failure to neutralize binding to eye muscle by pre-incubation of serum with membrane preparations from other tissues. Pre-incubation of thyrotrophin-binding-inhibiting immunoglobulin (TBII) with eye muscle membranes did not result in loss of thyroid stimulating hormone (TSH) binding inhibition. There was no correlation of ophthalmic immunoglobulins with microsomal antibody, thyroglobulin antibody, or TBII in serum of patients with Graves' disease. ${ }^{14}$

While thyroid-stimulating antibody (TSAb) is responsible for the hyperthyroidism of Graves' disease it does not appear to cause the ophthalmopathy. There is a dissociation clinically between GO and hyperthyroidism, ${ }^{15}$ a lack of correlation between long-acting thyroid stimulator (LATS), TBII, or TSAb activity and eye disease $\mathrm{e}^{16}$, a poor correlation between TBII and ophthalmic immunoglobulins, and pre-incubation of LATS or TBII with eye muscle membranes or other 
retro-orbital antigens does not neutralize the thyroid binding activity. ${ }^{17,18}$

Circulating immune complexes have been found in some patients with Graves' disease but are probably unrelated to ophthalmopathy. ${ }^{19,20}$ Immune complexes of thyroglobulin/thyroglobulin antibody have been reported to bind to eye muscle ${ }^{21}$ and local immune complex damage has been suggested as a possible mechanism for eye muscle disease. ${ }^{22}$ However, subsequent work has shown no correlation of thyroglobulin or its immune complexes with ophthalmopathy, ${ }^{23}$ and no correlation of thyroglobulin antibodies with ophthalmic immunoglobulins. ${ }^{14}$

The presence of both cell-mediated and humoral immune responses is therefore well established in GO but this does not explain the precise mechanisms causing the tissue damage resulting in ophthalmopathy.

Reports of uncontrolled hyper- or hypothyroidism leading to an exacerbation of the eye disease raise the possibility that hormonal factors may be involved in the pathogenesis. It is possible that thyroid hormones, TSH or even other hormones play a part in the development of $\mathrm{GO}$ by a direct effect on orbital tissue or on immune response cells although as yet the evidence for this is not convincing.

An understanding of the pathogenesis is important in order to develop a rational and effective approach to treatment which presently consists of local measures to relieve symptoms, correction of thyroid over- or under-activity, suppression of immune response cells or removal of immune complexes or antibodies, and surgical decompression of the orbit.

Hyper- or hypothyroidism should be corrected as quickly as possible, with the avoidance of swings from one to the other as this may be detrimental to the eyes. If thyroid hormones do have any effect then maintenance of euthyroidism is clearly important. The main thrust of treatment is directed at the immune system and both drugs to suppress $T$ cells and plasma exchange to remove antibodies and immune complexes have been used. There is little doubt that steroids given in large doses are effective,${ }^{24}$ particularly in those in whom the symptoms are of recent onset, but high doses are necessary and the condition often relapses when the dose is reduced. Cyclophosphamide

\section{References}

1. Bloomfield, G.W., Eckert, H., Fisher, M. et al. Treatment of thyrotoxicosis with radio-iodine: a review of 500 cases. $\mathrm{Br}$ Med J 1959, i: 63-74.

2. Volpe, R. Autoimmunity in thyroid disease. Monogr Endocrinol 1981, 20: 19-111.

3. Gorman, C.A. Temporal relationship between onset of Graves' ophthalmopathy and diagnosis of thyrotoxicosis. Mayo Clin Proc 1983, 58: 515-519. and azathioprine have been generally less effective, $\stackrel{\varrho}{3}$ although a current case report describes a dramatic $\stackrel{\mathbb{Q}}{\Omega}$ response to a combination of cyclophosphamide and $C$ orbital irradiation. ${ }^{25}$ Cyclosporin-A may be of some $\vec{F}$ value where the condition is severe and of recent $\stackrel{\text { s }}{\rightarrow}$ onset $^{11}$ although the response is rather variable. 을 Plasma exchange also produces variable responses. ${ }^{26,27} \frac{\overline{\bar{O}}}{\overline{\bar{N}}}$

There is no definite evidence that antithyroid drugs $\frac{\bar{s}}{\vec{D}}$ alone have any effect on the eyes but propylthiouracil $\stackrel{\Phi}{\varrho}$ followed by ablative doses of radio-iodine and hypothyroidism has led to some improvement in certain $\overrightarrow{0}$ cases. ${ }^{28}$ Thyroidectomy is considered effective by some $^{29}$ but others do not agree. After radio-iodine $\vec{\omega}$ treatment ophthalmopathy sometimes becomes $\stackrel{\Omega}{\circ}$ worse $^{30}$ and it is tempting to speculate that this relates 8 to an increase in circulating antibodies to thyroidal $\underline{3}$. antigens that commonly occurs 3 to 6 months after os radio-iodine therapy.

Indications for orbital decompression are: decreas- $\frac{\vec{\omega}}{\omega}$ ing visual acuity; impaired function of the orbital $\omega$ contents; and the appearance of the eye. The most $\vec{\Delta}$

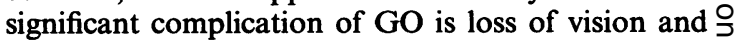
decreasing visual acuity, papilloedema, and increasing $\vec{c}$ visual field defects despite medical treatment are $C_{\mathbb{D}}$ unquestioned indications for orbital decompression. $\frac{D}{\mathbb{D}}$ The more common indications for surgery relate to $\frac{\mathbb{D}}{3}$ function of the eye such as the inability to close the lids which causes drying of the cornea with pain, corne@ ulceration, and possible perforation. Keratiti, $\oplus$ chemosis and lid oedema are also relative indicatio for surgery. Finally the cosmetic appearance of the eyes, which can be extremely distressing, must be considered as a further indication for surgery. Orbital decompression, however, can result in significant complications and does not attack the disease process. $\triangleright$ Surgery therefore should be considered only as symp- $\stackrel{2}{\longrightarrow}$ tomatically palliative or rehabilitative therapy and careful selection of appropriate patients is essential.

Whenever the pathophysiology of a disease is incompletely understood, treatment tends to be mul-tifaceted and directed at the alleviation of symptoms rather than the resolution of the disease. This is true of GO for which the treatment is still generally unsatisfactory and improvements are likely to occur only when the pathogenesis is more clearly understood.

4. Adams, D.D., Adams, Y.J., Knight, J.G. et al. On the nature of genes influencing the prevalence of Graves' $N$ disease. Life Sci 1983, 31: 3-13.

5. Tunbridge, W.M.G.T., Evered, D.C., Hall, R. \& Appleton, D $\omega$ The spectrum of thyroid disease in a community: the Whickham survey. Clin Endocrinol 1977, 7: 481-493.

6. Sugrue, D., McEvoy, M., Feely, J. \& Drury, M:I. Hyper- $\mathbb{D}$ thyroidism in the land of Graves: Results of treatment by : 远 
surgery, radioiodine, and carbimazole in 837 cases. $Q J$ Med 1980, 193, 51-61.

7. Farid, N.R., Sampson, L., Noel, E.P. et al. A study of human leukocyte D locus related antigens in Graves' disease. J Clin Invest 1979, 63: 108-113.

8. Mahieu, R. \& Winand, R. Demonstration of delayed hypersensitivity in retrobulbar and thyroid tissues in human exophthalmos. J Clin Endocrinol Metab 1972, 34: 1090-1092.

9. Munro, R.E., Lamki, L., Row, V.V. \& Volpe, R. Cellmediated immunity in the exophthalmos of Graves' disease as demonstrated by the migration inhibiton factor (MIF) test. J Clin Endocrinol Metab 1973, 37: 286-292.

10. Mullin, B.R., Levinson, R.E., Friedman, A. et al. Delayed hypersensitivity in Graves' disease and exophthalmos: identification of thyroglobulin in normal human orbital muscle. Endocrinology 1977, 100: 351-366.

11. Weetman, A.P., McGregor, A.M., Ludgate, M. \& Hall, R. Cyclosporin improves Graves' ophthalmopathy. Lancet 1983, ii: 486-489.

12. Blau, H.M., Kaplan, I, Tao, T.W. \& Kriss, J.P. Thyroglobulin independent cell-mediated cytotoxicity of human eye muscle cells in tissue culture by lymphocytes of a patient with Graves' ophthalmopathy. Life Sci 1983, 32: $45-53$.

13. Sisson, J.C., Kothary, P. \& Kirchick, H. The effect of lymphoctyes, sera, and long-acting thyroid stimulator from patients with Graves' disease on retrobulbar fibroblasts. J Clin Endocrinol Metab 1971, 37: 17-24.

14. Atkinson, S., Holcombe, M. \& Kendall-Taylor, P. Ophthalmic immunoglobulin in patients with Graves' ophthalmopathy. Lancet 1984, ii: 374-376.

15. Solomon, D.H., Chopra, I.J., Chopra, U. \& Smith, F.J. Identification of subgroups of euthyroid Graves' ophthalmopathy. N Engl J Med 1977, 296: 181-186.

16. McKenzie, J.M. \& McCullagh, E.P. Observations against a causal relationship between the long-acting thyroid stimulator and ophthalmopathy in Graves' disease. J Clin Endocrinol 1968, 28: $1177-1182$.

17. Shllinglaw, J. \& Utiger, R.D. Failure of retro-orbital tissue to neutralise the biological activity of the longacting thyroid stimulator. J Clin Endocrinol Metab 1968, 28: 1069-1070.

18. Atkinson, S., Holcombe, M., Taylor, J. \& Kendall-
Taylor, P. The relationship of ophthalmic IgG to TBII and other thyroid antibodies. Ann Endocrinol (Paris) 1984, 45: A8.

19. Takeda, Y. \& Kriss, J.P. Radiometric measurement of thyroglobulin-antithyroglobulin immune complexes in human serum. J Clin Endocrinol Metab 1977, 44: 46-55.

20. Brohee, D., Delespesse, G., Debisschop, M.J. \& Bonnyns, $M$. Circulating immune complexes in various thyroid diseases. Clin Exp Immunol 1979, 36: 379-383.

21. Konishi, J., Herman, M.M. \& Kriss, J.P. Binding of thyroglobulin and thyroglobulin-antithyroglobulin immune complex to extra ocular muscle membrane. Endocrinology 1974, 95: 434-446.

22. Kriss, J.P., Konishi, J. \& Herman, M. Studies in the pathogenesis of Graves' ophthalmopathy. Recent Prog Horm Res 1975, 31: 533-566.

23. Feldt-Rasmussen, U., Kemp, A., Bech, K., Madsen, S.N. \& Date, J. Serum thyroglobulin; its autoantibody and thyroid stimulating antibodies in the endocrine exophthalmos. Acta Endocrinol 1981, 96: $192-$ 198.

24. Werner, S.C. Prednisone in emergency treatment of malignant exophthalmos. Lancet 1966, 1004-1007.

25. Teoh, R. \& Woo, J. Combined irradiation and low dose cyclophosphamide in the treatment of Graves' ophthalmopathy, Postgrad Med J 1987, 63: 777-779.

26. Kelly, W., Longson, D., Smithard, D., et al. An evaluation of plasma exchange for Graves' ophthalmopathy. Clin Endocrinol 1983, 18: 485-493.

27. Glinoer, D., Schrooyen, M., Sand, G. \& Winand, R. Beneficial effects of plasma exchange for severe Graves' ophthalmopathy. Ann d'Endocrinol (Paris) 1984, 45: A10.

28. Gwinup, G., Elias, A.N. \& Ascher, M.S. Effect on exophthalmos of various methods of treatment of Graves' disease. JAMA 1982, 247: 2135-2138.

29. Catz, B. Total thyroidectomy and radioactive iodine ablation for the treatment of progressive ophthalmopathy of Graves' disease. Rev Port Ter Med 1974, 8: 88-92.

30. Kriss, J.P., Pleshakov, V., Rosenblum, Al., et al. Studies on the pathogenesis of the ophthalmopathy of Graves' disease. Studies on the pathogenesis os the ophthalmopathy of Graves' disease. J Clin Endocrinol 1967, 27: 582-593. 